DOI https://doi.org/10.30525/978-9934-26-008-7.2-16

\title{
LOSS OF CHARGED PARTICLES AS A CRITERION FOR THE FORMATION OF A HIGHLY CONDUCTIVE CHANNEL FOR THE PROTECTION OF ELECTRONIC EQUIPMENT
}

\author{
Iasechko M. M., Smyrnova I. M.
}

\section{INTRODUCTION}

The effect of EMR on weakly ionized air in the holes (waveguide antenna inputs) leads to an increase in the concentration of charged particles. This, under certain conditions, will lead to the appearance of an artificially created highly conducting channel, which is an electrical breakdown of the air gap between the hole walls or in the antenna waveguides.

The development of the breakdown to create a highly conductive channel is determined by many factors. These include the parameters of electromagnetic radiation (pulse duration of electromagnetic radiation, pulse repetition frequency and their energy), as well as physical processes occurring at different stages of the breakdown, and determine the conditions for its occurrence. In other words, the creation of a highly conductive channel is determined by the process of interaction of the current control device (CCD) EMR with a gas-plasma medium.

The formation of a highly conductive channel is necessary for the closure and subsequent removal of EMR from the REM.

The works of scientists Joffe, Rogowski, MacDonald, Raiser, Omarov, Rukhadze, Hecker, Loeb, Vazhov, Lavrinovich, Townsend, Dyakov, Bobrov, Sorokin and others widely presented the theory of breakdown of various gases, atmospheres, condensed dielectric media. Physical and mathematical and general mathematical models of gas breakdown are given. Breakdown criteria are proposed, breakdown conditions are investigated, in particular, the electron concentration at which breakdown occurs. Various types of electrical discharges in the fields of different frequency ranges are considered. The results of numerical simulation of breakdown for one and two-dimensional cases are presented, which do not fully take into account the physical processes 
that affect the concentration of electrons and ions in the discharge gap when exposed to CCD EMR.

General information on expressions for determining diffusion coefficients, sticking, electron temperature variation, scattering and recombination cross sections exist in the hydrodynamic approximation, but they were obtained without taking into account the parameters of the EMR effect.

Analysis of the main provisions of the theory of breakdown in gaseous media has shown that one of the promising places in it is solving the problem of meeting the necessary requirements for a gaseous medium, namely, a sufficient concentration of particles when exposed to CCD EMR, to ensure guaranteed breakdown and, accordingly, the REM protection.

\section{Main material}

According to a formalized mathematical model describing the CCD EMR interaction with a weakly ionized air medium, which includes a plasma, plasma avalanche and plasma streamer stage, the condition for the formation of a highly conductive state in the discharge gap is to create a concentration of charged particles $n$ exceeding the critical one $n \geq n_{c r}$.

Providing such a concentration depends on the following:

1) geometrical parameters of the discharge gap (the linear dimensions of the holes, slots, cable entry channels) and pressure values;

2 ) intensity of the preliminary ionization source in the discharge gap;

3) processes occurring during the interaction of EMR with a preionized air through the use of an ionization source.

Collective processes in the discharge gap of a hole determine the state of the electronic subsystem and affect the EMR, which, in turn, determines this state. At the same time, due to the interaction with the plasma medium, an EMR pulse is deformed, which influences the formation of a highly conducting state. On this basis, the solution of the problem of determining the conditions for the occurrence of a critical concentration of electrons $n_{c r}$ in an air medium (changes in the leading state) depending on the kinetic processes occurring during the formation of the plasma medium, taking into account its interaction with electromagnetic radiation, first of all requires the installation of the interdependence of the main macroscopic properties of slightly ionized environment in the holes (waveguide antenna inputs) REM and EMR parameters. This leads to the necessity of splitting this task into a series 
of subtasks. The paper proposes to consider one of these subtasks, which is to study the effect of EMR on the previously ionized medium in the discharge gap.

Solution of this subtask is associated with ionization and kinetic processes occurring in the discharge gap of a pre-ionized air environment under the influence of CCD EMR and cause the appearance of a number of effects not observed in fields of moderate intensity. First of all, it concerns the following:

- during the breakdown of a gas by an electromagnetic pulse or an intense microwave pulse, a high ionization rate substantially changes the space-time pattern of discharge development;

- effect of kinetic processes on the parameters of a nanosecond discharge, which determine the instabilities of the discharge plasma, its decomposition, absorption capacity and chemical activity.

These processes lead to the loss of a part of charged particles, besides the electric field leads to a decrease in the rate of diffusion flow, which leads to a decrease in the electrical conductivity of the medium. These phenomena lead to an increase in the time of occurrence of the highly conductive state in the discharge gap of the hole necessary for the implementation of the EMR circuit.

Depending on the magnitude of these parameters, first of all, the ratios of pressure and the size of the discharge gap, it is possible to describe the occurrence of a highly conductive state using the Townsend and plasma-streamer breakdown models ${ }^{1}$. But the theory of Townsend breakdown is valid for small discharge gaps (less than $1 \mathrm{sm}$ ) at $p d \leq 200$ torr $\cdot s m$ and without a noticeable overvoltage in the discharge gap. The latter circumstance makes it possible to ignore the influence of the space charge on the development of the discharge. This type of breakdown can also occur at large values $p d \geq 1000$ torr $\cdot s m$ in the presence of overvoltage.

Therefore, the direct use of the Townsend breakdown theory to describe the functioning of a protective device is impossible due to the fact that the breakdown description is carried out for conditions of limited volume and occurs not only under the influence of pulsed EMR, but also due to the periodization by $\alpha$-particles of the discharge gap. The last influence factor leads to the evolution of the distribution function and the flux of charged

1 Artsimovich L.A., Sagdeev R.Z. Plasma physics for physicists. Moscow. 1979. $320 \mathrm{p}$. 
particles in the phase space and to the appearance of a non-equilibrium state of the electronic subsystem. In turn, this leads to the attenuation of the energy of the electromagnetic radiation in the ionized medium of the discharge gap, a decrease in the ionization and, accordingly, to an increase in the time of breakdown occurrence. In addition, the effect of electromagnetic radiation leads to an uneven increase in the concentration of particles over time, which is determined by the length of the pulse front. As a result, the well-known breakdown criterion, which determines the time of formation of a highly conductive state in the discharge gap, as well as the magnitude of the breakdown voltage, taking into account the parameters of electromagnetic radiation, needs to be refined.

The space charge field under conditions of extended discharge gaps (up to $7 \mathrm{sm}$ ) at atmospheric pressure begins to significantly affect the discharge and leads to the appearance of plasma-streamer breakdown ${ }^{23}$.

Three stages of the formation of a highly conductive channel to ensure the guaranteed protection of large holes, slots or antenna waveguide inputs through plasma-streamer breakdown require the determination of the boundary conditions for the transition from one stage to the next. Thus, according to the model of a highly conductive channel in the form of a three-stage structure $^{4}$, the macroscopic parameters of the plasma medium are determined depending on the EMR parameters.

\section{Results of the analysis of the influence of the electric field on the loss of charged particles}

In the absence of external electromagnetic radiation in the air environment of the hole ( $d \geq 3,8 \mathrm{sm}$ ), the quasistationary equilibrium state of the electronic subsystem is established. This is due to the fact that the size of the hole exceeds the length of the free path of $\alpha$-particles in air.

The distribution function of charged particles in the discharge gap is the initial one for determining the main macroscopic parameters of

${ }^{2}$ Ropy A.N., Starik A.M., Shutov K.K. Microwave protective device. Moscow. 1993. 128 p.

${ }^{3}$ Krizny A.V., Vorobyov O.M., Sotnikov O.M. Designing the structure of the material of the protective screens of radio-electronic means of arms and military equipment from the effects of powerful electromagnetic radiation pulse duration. Trudy Universitetu. 2013. No. 6 (120). Pp. 187-191.

${ }^{4}$ Mykhailov V. Providing of firmness of side digital calculable machines to influence of supershort electromagnetic impulses. Moscow. 2009. 24 p. 
ionized air. But under the influence of external EMR there will be a change in the state of its electronic subsystem. Depending on the power of electromagnetic radiation, frequency and length of the pulse, these processes will be different and differently determine the conditions for the implementation of the REM protection.

Since the formation of a highly conductive channel for discharge is determined by the processes of generation of charged particles and their losses due to diffusion, adhesion, change in electron temperature, and recombination, we consider each of these processes separately, depending on the parameters of electromagnetic radiation.

Let's suppose that a pre-ionized air environment is affected by a weak electric field $\mathrm{E}(\mathrm{r})$. In this case, the force that affects the charged particles is equal to $F=-\Delta E$. Electrons under the action of force $F$ begin to move along its direction. As a result, the distribution function evolves, which is no longer Maxwellian.

It is known that under conditions when the distribution function differs little from the equilibrium $f(r, v, t)=f_{0}(v)+f_{1}(r, v, t)$ collision integral $I_{3}(r, v, t)=\left(\frac{d f}{d t}\right)_{3}$ can be replaced by a model collision integral in the $\tau$-approximation [4]:

$$
\left.\frac{\partial \mathrm{f}}{\partial \mathrm{t}}\right|_{3}=\frac{\mathrm{f}_{0}(\mathrm{v})-\mathrm{f}_{1}(\mathrm{r}, \mathrm{v}, \mathrm{t})}{\tau}=\frac{\mathrm{f}_{0}-\mathrm{f}_{1}(\mathrm{r}, \mathrm{v}, \mathrm{t})}{\tau},
$$

Electrons can collide with neutrals during the movement. As a result, they reach a certain velocity value. Thus, the electric field of the working signals causes a current in the weakly ionized air medium of the waveguide. The ratio of the magnitude of the current to the magnitude of the electric field determines the magnitude of the conductivity of the medium.

\section{The influence of the electric field on the transfer coefficients of the pre-ionized medium in the opening of the box-screen}

To calculate the change in the electron distribution function as a result of the electric field of the signal, let's present the electron distribution function as follows:

$$
\mathrm{f}=\mathrm{f}_{0}+\mathrm{f}_{1},
$$

where $f_{0}>>f_{1}$. 
Taking into account the influence of an electrical signal, the electron distribution function is represented by the following relation:

$$
\mathrm{f}=\mathrm{f}_{0}+\frac{\mathrm{eE}}{\mathrm{m}_{\mathrm{e}} \mathrm{v}_{\mathrm{me}}} \frac{\partial \mathrm{f}_{0}}{\partial \mathrm{V}},
$$

where $v_{\mathrm{me}}=\frac{1}{\tau}-$ the frequency of collisions of electrons with neutrals with the transfer of momentum;

e - electron charge; $\mathrm{m}_{\mathrm{e}}-$ electron mass.

Taking into account the expression (2), the conduction current density in the $\mathrm{z}$ direction will be represented in the following version:

$$
J=-\operatorname{en} \int \mathrm{vf} \partial \mathrm{v}=-\frac{\mathrm{e}^{2} \mathrm{E}_{\mathrm{z}}}{\mathrm{m}_{\mathrm{e}} \mathrm{v}_{\mathrm{me}}} \mathrm{n} \int \mathrm{v}_{\mathrm{z}} \frac{\partial \mathrm{f}_{0}}{\partial \mathrm{v}_{\mathrm{z}}} \partial \mathrm{v} .
$$

After integration (3) in parts, taking into account the valuation $\int \mathrm{f} \partial \mathrm{v}=1$, let's obtain:

$$
\mathrm{J}_{\mathrm{z}}=\frac{\mathrm{ne}^{2} \mathrm{E}_{\mathrm{z}}}{\mathrm{m}_{\mathrm{e}} v_{\mathrm{me}}} .
$$

From (4) let's obtain the expression for the conductivity of the preionized air medium in terms of the frequency $v_{m e}$ of collisions of electrons with neutrals:

$$
\sigma=\frac{n e^{2}}{m_{\mathrm{e}} v_{\mathrm{me}}}=\frac{\omega_{\mathrm{p}}^{2}}{4 \pi v_{\mathrm{me}}} .
$$

According to (5), the conductivity of the pre-weakly ionized air medium in the opening of the REM box-screen is determined by the plasma frequency and the frequency of collisions of electrons with neutrals $\left(\omega_{\mathrm{p}} \ll v_{\mathrm{me}}\right)$ and is small.

This means that the previous ionization of the air environment for the protection of the REM antenna waveguide using the source of $\alpha$-particles does not affect the passage of the working signal, is always small.

Under the influence of an ionization source, as well as an electric field, an ion concentration gradient arises, which, due to collisions, involves electrons. As a result, the conductivity of the ionized air in the REM holes or waveguides changes. 
Let's analyze the effect of diffusion of particles on the conductivity of the discharge gap.

The diffusion rate is determined by the diffusion coefficient, which can be represented through the stream of particles by the following expression ${ }^{5}$ :

$$
\Pi_{\mathrm{a}}=\overline{\mathrm{n}}_{\mathrm{a}} \int \mathrm{vf}_{\mathrm{a}}(\mathrm{v}) \mathrm{dn}=-\mathrm{D} \nabla \mathrm{n},
$$

where $\overline{\mathrm{n}}_{\mathrm{a}}=\mathrm{n}_{\mathrm{a}} / \mathrm{v}$ - the average density of particles of $a$ sort.

At small pressure gradients, which arise due to the heating of the ionized air by the electric field and lead to the appearance of particle concentration gradients [3]. Their distribution function can be represented as an expansion according to the type of definition of electrical conductivity, where the particle flux distribution function $f_{a 1}$ $(\mathrm{x}, \mathrm{v})$, due to the particle concentration gradient, is considered small compared to the local Maxwell distribution function $\mathrm{f}_{\mathrm{ao}}(\mathrm{x}, \mathrm{v})$.

In our case, the concentration gradient of particles due to the use of an ionization source can be considered constant. This provides a stationary state, which is described by a kinetic equation with a model collision integral in the $\tau$-approximation. As a result, taking into account the distribution function of the flux of particles $f_{a 1}(x, v)$, the frequency of collisions of particles of a sort with neutrals $v_{\text {ma }}$ in the $\mathrm{X}$ direction can be written:

$$
\Pi_{\mathrm{x}}=-\frac{1}{v_{\mathrm{ma}}} \frac{\mathrm{d}}{\mathrm{dx}} \int \overline{\mathrm{n}}_{\mathrm{a}} \mathrm{v}_{\mathrm{x}}^{2} \mathrm{f}_{\mathrm{a}_{0}} \mathrm{dv}=-\frac{1}{v_{\mathrm{ma}}} \frac{\mathrm{d}}{\mathrm{dx}} \frac{\mathrm{nkT}}{\mathrm{m}_{\mathrm{a}}},
$$

where $\mathrm{k}$ - the Boltzmann constant.

Taking into account (6), the expression for the diffusion coefficient of the particles of the a sort will be:

$$
\mathrm{D}_{\mathrm{a}}=\frac{\mathrm{kT}_{\mathrm{a}}}{\mathrm{m}_{\mathrm{a}} \mathrm{v}_{\mathrm{ma}}} .
$$

By analogy, it is possible to write expressions for the diffusion coefficients of electrons and ions:

$$
\mathrm{D}_{\mathrm{e}}=\frac{\mathrm{kT}}{\mathrm{m}_{\mathrm{e}} v_{\mathrm{me}}}, \mathrm{D}_{\mathrm{i}}=\frac{\mathrm{kT}}{\mathrm{m}_{\mathrm{i}} v_{\mathrm{mi}}},
$$

${ }^{5}$ Starostenko V.V., Grigoriev V.V., Taran E.P. The impact of electromagnetic fields on the stability of the IC. Mat. 6th Int. Crimean Microwave Conf. (Sevastopol, Sept. 16-19. 1996). Sevastopol. 1996. Pp. 188-191. 
where $v_{\mathrm{me}}, v_{\mathrm{mi}}$ - frequency of collisions of electrons and ions with neutrals, respectively.

Due to the smaller electron mass, diffusion will be faster than ions. This will result in a space charge and an associated electric field. As a result, this field will prevent electrons from escaping and help the ions to leave the ionized medium. In turn, a steady state will arise when the fluxes of ions and electrons become equal. This phenomenon resulting from the self-consistent process is called ambipolar diffusion ${ }^{6}$.

The ambipolar diffusion coefficient can be found by equating the fluxes of ions and electrons. As a result, the expression for the resultant flux of particles of any kind can be written in terms of the ambipolar diffusion coefficient $\mathrm{D}_{\mathrm{a}}$ :

$$
\mathrm{D}_{\mathrm{a}}=\frac{\mathrm{k}_{\mathrm{pi}} \mathrm{D}_{\mathrm{e}}-\mathrm{k}_{\mathrm{pe}} \mathrm{D}_{\mathrm{i}}}{\mathrm{k}_{\mathrm{pi}}-\mathrm{k}_{\mathrm{pe}}} \nabla \mathrm{n},
$$

where $\mathrm{k}_{\mathrm{pi}}, \mathrm{k}_{\mathrm{pe}}-$ mobility coefficients of ions and electrons.

Taking into account the fact that the diffusion and mobility coefficients are related by the relation (2), let's present the expression for ambipolar diffusion coefficient (10) due to temperature and diffusion coefficients by the following relation:

$$
\mathrm{D}_{\mathrm{a}}=\frac{\left(\mathrm{T}_{\mathrm{e}}+\mathrm{T}_{\mathrm{i}}\right) \mathrm{D}_{\mathrm{i}} \mathrm{D}_{\mathrm{e}}}{\mathrm{T}_{\mathrm{i}} \mathrm{D}_{\mathrm{e}}+\mathrm{T}_{\mathrm{e}} \mathrm{D}_{\mathrm{i}}} .
$$

Let's take into account $D_{i}<<D_{e}$ that if expression (11) takes the form:

$$
\mathrm{D}_{\mathrm{a}} \approx \mathrm{D}_{\mathrm{i}}\left(1+\frac{\mathrm{T}_{\mathrm{e}}}{\mathrm{T}_{\mathrm{i}}}\right) .
$$

For the case $T_{e} \approx T_{i}:$

$$
\mathrm{D}_{\mathrm{a}} \approx 2 \mathrm{D}_{\mathrm{i}} \text {. }
$$

Thus, the diffusion coefficient for a weakly ionized medium is almost twice the ion diffusion coefficient, which reduces the electronic conductivity of the medium and, accordingly, negatively affects the implementation of the EMR circuit when it interacts with the holes, slots of the box-screens and cable inlets of the REM.

${ }^{6}$ Dobykin V.D., Kupriyanov A.I., Ponomarev V.G., Shustov L.N. Electronic Warfare. Power failure of electronic systems. Moscow. 2007. 487 p. 
Taking into account the diffusion coefficient of ions, the particle concentration at time $t$ at a distance $r$ from the ionization source in the three-dimensional case is determined by the relation ${ }^{7}$ :

$$
\mathrm{n}=\mathrm{n}_{0} \frac{1}{(4 \pi \mathrm{Dt})^{\frac{3}{2}}} \mathrm{e} \frac{-\mathrm{r}^{2}}{4 \mathrm{Dt}} .
$$

Then, taking into account (8), (9), (12), expression (13) in the $\mathrm{X}$ direction is written down:

$$
\mathrm{n}=\mathrm{n}_{0} \frac{1}{\left(\Omega \frac{\mathrm{kT}}{\mathrm{m}_{\mathrm{i}} v_{\mathrm{mi}}}\left(1+\frac{\mathrm{T}_{\mathrm{e}}}{\mathrm{T}_{\mathrm{i}}}\right) \mathrm{t}\right)^{\frac{3}{2}}} \mathrm{e}^{-\frac{\mathrm{x}^{2}}{4 \frac{k \mathrm{~T}_{\mathrm{i}}}{\mathrm{m}_{\mathrm{i}} \mathrm{vin}_{\mathrm{mi}}}\left(1+\frac{\mathrm{T}_{\mathrm{e}}}{\mathrm{T}_{\mathrm{i}}}\right) \mathrm{t}}} .
$$

Expression (14) determines the change in the concentration of ions in a solid angle $\Omega$ with regard to their scattering.

Let's take into account that the diffusion coefficient of $D$ ions is related to the mobility coefficient $\mathrm{k}_{\mathrm{p}}$ by a ratio that determines the influence of the amount of air pressure at which this process occurs ${ }^{8}$ :

$$
\mathrm{k}_{\mathrm{p}}=\frac{\mathrm{neD}}{\mathrm{p}}
$$

where $\mathrm{n}$ is the number of molecules in $1 \mathrm{~cm}^{2}$ at pressure $\mathrm{p}$.

At a significant electric field, the drift velocity of the ion is much higher than the thermal velocity of the atoms. Therefore, atoms can be considered fixed. After each recharge, the ion stops, then picks up velocity by interacting with an electric field.

From the equation of motion of an ion, depending on the magnitude of the field $\mathrm{E}$, let's find the relative number of ions with a relative collision velocity, which corresponds to the ion velocity along the field:

$$
f\left(v_{x}\right)=A\left[-\int_{0}^{v_{x}} n v_{x}^{\prime} \sigma_{\text {peз }}\left(v_{x}^{\prime}\right) \frac{m_{i}}{e E} d v_{x}^{\prime}\right],
$$

7 Baliuk N., Kechyev L., Stepanov P. Powerful electromagnetic impulse: affecting electronic facilities and methods of defence. Moscow. 2007. 478 p.

${ }^{8}$ Magda I.I., Bludov S.P., Gadetski N.P., et al. Studies on electronic device degradation phenomena under interference of pulsed-power electromagnetic fields. 3-rd Int. Crimean Conf. "UNF Tech. and satellite reception". Sevastopol. 1993. Pp. 523-526. 
where A - a constant determined from the normalization condition of the distribution function.

Assuming that the resonance charge exchange cross section does not depend on the collision rate, from expression (16) let's find the coefficient of ion mobility at significant external field voltages:

$$
\mathrm{k}_{\mathrm{pi}}=\sqrt{\frac{2 \mathrm{e}}{\pi \mathrm{m}_{\mathrm{i}} \mathrm{n}_{\mathrm{i}} \mathrm{E} \sigma_{r e m}}} .
$$

The coefficient of ion mobility for small values of the voltage of the REM operation signal can be found from the well-known ChapmanEnskog expression ${ }^{9}$ :

$$
\mathrm{k}_{\mathrm{pi}}=\frac{0,33 \mathrm{e}}{\mathrm{n}_{\mathrm{i}} \sqrt{\mathrm{m}_{\mathrm{i}} \mathrm{T}_{\mathrm{i}} \sigma_{\text {pes }}}\left(2,1 \sqrt{\frac{2 \mathrm{~T}_{\mathrm{i}}}{\mathrm{m}_{\mathrm{i}}}}\right)} .
$$

Analysis of expressions (17), (18) shows that the mobility of ions with a high electric field voltage depends inversely on the voltage of this field and does not depend on the electric field with a low voltage, which characterizes the REM operation signal.

Thus, EMR has a significant impact on the mobility of charged particles and, accordingly, on the formation of a highly conductive channel for the implementation of the EMRP circuit in the REM protection facilities.

According to (6), (7), (14), diffuse processes are determined by electron temperature, which in a weakly ionized medium under the influence of an external field differs significantly from air temperature. Let's determine the effect of an alternating electric field on the electron temperature of a weakly ionized air medium in the opening.

Let's suppose that the electric field $\mathrm{E}(\mathrm{t})$ varies according to a harmonic law $\mathrm{E}(\mathrm{t})=\mathrm{E} \cos \omega \mathrm{t}$.

Then the kinetic equation (19) let's write in a simplified form:

$$
\frac{\partial \mathrm{f}}{\partial \mathrm{t}}+\frac{\mathrm{eE}}{\mathrm{m}_{\mathrm{e}}} \cos \omega \mathrm{t} \frac{\partial \mathrm{f}}{\partial \mathrm{V}_{\mathrm{e}}}=\mathrm{I}_{3}(\mathrm{f}),
$$

Let's write the system of equations for $f_{0}$ and $f_{1}$

${ }^{9}$ Krutov A., Mitmen V., Rebrov A. Protector of the small power level. Materials proc. of 12th International Crimean Conference "Microwave equipment". Sevastopol. 2002. Pp. 93-94. 


$$
\left\{\begin{array}{l}
\frac{\partial \mathrm{f}_{0}}{\partial \mathrm{t}}+\mathrm{f}_{1} \frac{e E \cos \omega \mathrm{t}}{3 \mathrm{~m}_{\mathrm{e}} \mathrm{V}_{\mathrm{e}}^{2}} \frac{\partial \mathrm{V}_{\mathrm{e}}^{3} \mathrm{f}_{1}}{\partial \mathrm{V}_{\mathrm{e}}}=\mathrm{I}_{3}\left(\mathrm{f}_{0}\right) \\
\frac{\partial \mathrm{f}_{1}}{\partial \mathrm{t}}+\frac{\mathrm{eE \operatorname {cos } \omega \mathrm { t }}}{\mathrm{m}_{\mathrm{e}} \mathrm{V}_{\mathrm{e}}} \frac{\partial \mathrm{f}_{0}}{\partial \mathrm{V}_{\mathrm{e}}}=-v_{e} \mathrm{f}_{1}
\end{array},\right.
$$

where $v_{e}-$ the frequency of elastic collisions.

For REM of $\mathrm{HF}$ and microwave ranges, the condition

$$
\omega \mathrm{t}>>1
$$

Then, if we take into account that the functions $f_{0}$ and $f_{1}$ depend on $\omega t$ the fact that their expansions in a Fourier series are:

$$
\mathrm{f}_{0}\left(\mathrm{~V}_{\mathrm{e}}, \mathrm{t}\right)=\sum_{\mathrm{n}} \mathrm{f}_{0, \mathrm{n}}\left(\mathrm{V}_{\mathrm{e}}\right) \mathrm{e}^{\mathrm{i} \omega \mathrm{nt}}, \mathrm{f}_{1}\left(\mathrm{~V}_{\mathrm{e}}, \mathrm{t}\right)=\sum_{\mathrm{n}} \mathrm{f}_{1, \mathrm{n}}\left(\mathrm{V}_{\mathrm{e}}\right) \mathrm{e}^{\mathrm{i} \omega \mathrm{nt}} .
$$

As a result, the system of equations (20) is rewritten as follows:

$$
\left\{\begin{array}{l}
\omega_{0, n}+\frac{e E}{6 \mathrm{mV}_{\mathrm{e}}^{2}} \frac{\mathrm{dV}_{\mathrm{e}}^{3}\left(\mathrm{f}_{1, \mathrm{n}+1}+\mathrm{f}_{1, \mathrm{n}-1}\right)}{\mathrm{dV}}=\mathrm{I}_{3}\left(\mathrm{f}_{0, \mathrm{n}}\right), \\
\mathrm{i \omega nf_{1, \textrm {n } }}+\frac{\mathrm{eE}}{2 \mathrm{mV}} \frac{\mathrm{d}\left(\mathrm{f}_{0, \mathrm{n}-1}+\mathrm{f}_{0, \mathrm{n}+1}\right)}{\mathrm{dV}}=v_{\Pi 1, \mathrm{f}} .
\end{array}\right.
$$

Assuming that the collision of electrons with atoms of ionized air is determined only by elastic collisions, the function $\mathrm{f}_{0}$ will be determined by the relation:

$$
f_{0}\left(V_{e}\right)=\operatorname{Aexp}\left(-\int_{0}^{V} \frac{m_{e} V_{e}}{T+\frac{e^{2} E^{2} m_{a}}{6 m_{e}^{2}\left(\omega^{2}+v_{e}^{2}\right)}} d V\right),
$$

where $\mathrm{A}$ - the valuation constant;

$\mathrm{m}_{\mathrm{a}}$ - the mass of the atom. 
According to the distribution function $\mathrm{f}_{1}(\mathrm{~V}, \mathrm{t})$ will be determined by the relation ${ }^{10}$ :

$$
\mathrm{f}_{1}(\mathrm{~V}, \mathrm{t})=-\frac{\mathrm{eE}}{\mathrm{m}_{\mathrm{e}}\left(\omega^{2}+v_{e}^{2}\right) \mathrm{V}_{\mathrm{e}}}\left(v_{e} \cos \omega \mathrm{t}+\sin \omega \mathrm{t}\right) \frac{\mathrm{df}_{0}}{\mathrm{dV}_{\mathrm{e}}} .
$$

Since in our case $\omega \gg v_{e}$, from (22) and (23) let's write the expression for the electron drift velocity:

$$
\mathrm{u}(\mathrm{t})=\frac{\mathrm{eE}}{3 \mathrm{~T}_{\mathrm{e}}}\left\langle\mathrm{V}_{\mathrm{e}}^{2} \frac{v_{e} \cos \omega \mathrm{t}}{\omega^{2}+v_{e}^{2}}\right\rangle,
$$

where $\langle\rangle-$. the operation of statistical averaging over the electron velocity.

By multiplying the energy balance equation for an individual electron, namely the magnitude of both parts by $\frac{\mathrm{mV}_{\mathrm{e}}^{2}}{2}$, further integration over the electron velocity and averaging over time, we get the expression:

$$
\overline{\frac{\partial}{\partial t}\left\langle\frac{m_{\mathrm{e}} V_{\mathrm{e}}^{2}}{2}\right\rangle}+\overline{\mathrm{eEU} \cos \omega \mathrm{t}}=\int \frac{\mathrm{m}_{\mathrm{e}} \mathrm{V}_{\mathrm{e}}^{2}}{2} \mathrm{I}_{\mathrm{ea}} \mathrm{dV}_{\mathrm{e}} .
$$

In view of (24) and let's obtain:

For the case taking into account the energy balance equation for an individual electron it is possible to write the system of equations for the distribution functions $\mathrm{f}_{0}$ and $\mathrm{f}_{1}$ in the form ${ }^{11}$ :

$$
\left\{\begin{array}{l}
\frac{\mathrm{eE}}{3 \mathrm{~m}_{\mathrm{e}}} \frac{\mathrm{df}_{\mathrm{l}} \mathrm{V}_{\mathrm{e}}^{3}}{\mathrm{~V}_{\mathrm{e}}^{2} \mathrm{dV}}=\mathrm{I}_{\mathrm{ae}}+\mathrm{I}_{\mathrm{ee}} \\
\frac{\mathrm{eE}}{\mathrm{m}_{\mathrm{e}}} \frac{\mathrm{df}_{0}}{\mathrm{dV}}=-v_{e} \mathrm{~V}_{\mathrm{e}} \mathrm{f}_{1}
\end{array} .\right.
$$

${ }^{10}$ Alybin V.G. Problems of creation of protective devices for microwave radar and communication. 12th Int. Crimean Conference "Microware Telecommunication Technology”. (Sevastopol, 9-13 September. 2002). Sevastopol. 2002. Pp. 15-21.

${ }^{11}$ Dobykin V.D., Kupriyanov A.I., Ponomarev V.G., Shustov L.N. Electronic Warfare. Power failure of electronic systems. Moscow. 2007. 487 p. 
In our case $v_{\mathrm{ee}} \sim \mathrm{I}_{\mathrm{ee}}>>\mathrm{I}_{\mathrm{ae}} \sim \frac{\mathrm{m}_{\mathrm{e}}}{\mathrm{m}_{\mathrm{a}}} v_{\mathrm{ea}}$. Therefore, from (27), by multiplying the first equation by the expression for the kinetic energy of electrons $\frac{\mathrm{mV}_{\mathrm{e}}^{2}}{2}=\mathrm{E}_{\mathrm{K}_{\mathrm{e}}}$ and further integration over velocity, let's obtain:

$$
\mathrm{eEu}=\int \frac{\mathrm{m}_{\mathrm{e}} \mathrm{V}_{\mathrm{e}}^{2}}{2} \mathrm{I}_{\mathrm{ea}} \mathrm{dV} \mathrm{V}_{\mathrm{e}}
$$

From this equation let's write:

$$
\mathrm{f}_{1}=\frac{\mathrm{eEf}_{0}}{v_{\pi} \mathrm{T}}
$$

As a result, the electron drift velocity will be determined by the expression:

$$
\mathrm{u}=\frac{\mathrm{eE}}{\mathrm{T}_{\mathrm{e}}}\left\langle\frac{\mathrm{V}_{\mathrm{e}}^{2}}{3 \mathrm{v}_{e}}\right\rangle .
$$

Considering the fact that for our case the frequency of elastic collisions is related to the frequency of thermal collisions $v_{T}$ by the expression:

$$
v_{e}=v_{\mathrm{T}}\left(\frac{\mathrm{m}_{\mathrm{e}} \mathrm{V}_{\mathrm{e}}^{2}}{2 \mathrm{~T}_{\mathrm{e}}}\right)^{\mathrm{n} / 2},
$$

obtain:

$$
v_{e}=v_{\mathrm{T}}\left(\frac{\mathrm{m}_{\mathrm{e}} \mathrm{V}_{\mathrm{e}}^{2}}{2 \mathrm{~T}_{\mathrm{e}}}\right)^{\mathrm{n} / 2}
$$

where $\mathrm{x}=\sqrt{\mathrm{m}_{\mathrm{e}} \mathrm{V}_{\mathrm{e}}^{2} / 2 \mathrm{~T}_{\mathrm{e}}}$.

Now, taking into account (28) it is possible to write the energy balance equation in the following form:

$$
-\left\langle\frac{\mathrm{m}_{\mathrm{e}} \mathrm{V}_{\mathrm{e}}^{2}}{2} \mathrm{I}_{\mathrm{ea}}\right\rangle=\frac{8}{\sqrt{\pi}} \frac{\mathrm{m}_{\mathrm{e}}}{\mathrm{m}_{\mathrm{a}}}\left(\mathrm{T}_{\mathrm{e}}-\mathrm{T}\right) v_{e} \Gamma\left(\frac{5+\mathrm{n}}{2}\right) .
$$


Taking into account that $\mathrm{eEu}=-\left\langle\frac{\mathrm{m}_{\mathrm{e}} \mathrm{V}_{\mathrm{e}}^{2}}{2} \mathrm{I}_{\mathrm{ea}}\right\rangle$.

Then from (29) let's obtain:

$$
\mathrm{T}_{\mathrm{e}}=\mathrm{T}+\mathrm{m}_{\mathrm{a}} \frac{\mathrm{e}^{2} \mathrm{E}^{2}}{3 \mathrm{~m}_{\mathrm{e}}^{2} v_{\mathrm{T}}^{2}} \frac{\Gamma((5-\mathrm{n}) / 2)}{\Gamma((5+\mathrm{n}) / 2)} .
$$

If $n=0$

$$
\mathrm{T}_{\mathrm{e}}=\mathrm{T}+\frac{\mathrm{m}_{\mathrm{a}} \mathrm{e}^{2} \mathrm{E}^{2}}{3 \mathrm{~m}_{\mathrm{e}}^{2} \mathrm{v}_{\mathrm{T}}^{2}}
$$

at $n=1$

$$
\mathrm{T}_{\mathrm{e}}=\mathrm{T}+\frac{\mathrm{m}_{\mathrm{a}} \mathrm{e}^{2} \mathrm{E}^{2}}{6 \mathrm{~m}_{\mathrm{e}}^{2} v_{\mathrm{T}}^{2}} .
$$

Taking into account (26), let's write the expression (32) for large field strengths:

$$
\mathrm{T}_{\mathrm{e}}=\mathrm{T}+\frac{\mathrm{m}_{\mathrm{a}}}{6}\left(\frac{\mathrm{eE}}{\mathrm{m}_{\mathrm{e}}}\right)^{2}\left\langle\frac{1}{\omega^{2}+v_{e}^{2}}\right\rangle .
$$

For our case $\left(\omega \gg v_{\mathrm{e}}\right)$ :

$$
\mathrm{T}_{\mathrm{e}}=\mathrm{T}+\frac{\mathrm{m}_{\mathrm{a}}}{6}\left(\frac{\mathrm{eE}}{\mathrm{m}_{\mathrm{e}} \omega}\right)^{2}
$$

From (34) it can be seen that at high electric field strength, the electron temperature of ionized air significantly exceeds the thermal energy of the particles, and the difference between electron and atomic temperatures depends on the frequency of the field.

Taking into account (34), expression (14) is written in the form: 


$$
\begin{aligned}
& \mathrm{n}(\mathrm{Q}, \mathrm{E}, \mathrm{x}, \mathrm{t})=\frac{\mathrm{n}_{0}(\mathrm{Q})}{\left(\mathrm{A}(\mathrm{E}) \Omega \frac{\mathrm{kT}_{\mathrm{i}}}{\mathrm{m}_{\mathrm{i} \mathrm{v}_{\mathrm{mi}}}}\left(\mathrm{t}-\mathrm{t}_{0}\right)\right)^{\frac{3}{2}}} \exp \left(v_{\mathrm{i}} \mathrm{t}-\mathrm{A}^{-1}(\mathrm{E}) \frac{\mathrm{x}^{2}+\left(\mathrm{x}-\mathrm{v}_{\mathrm{i}} \mathrm{t}_{\mathrm{d}}\right)^{2}}{\left.4 \frac{\mathrm{kT}_{\mathrm{i}}}{\mathrm{m}_{\mathrm{i} \mathrm{v}_{\mathrm{mi}}}\left(\mathrm{t}-\mathrm{t}_{0}\right)}\right)}\right. \\
& \text { where } A(\mathrm{E})=\left(1+\frac{\mathrm{T}+\frac{\mathrm{m}_{\mathrm{a}}}{6}\left(\frac{\mathrm{eE}}{\mathrm{m}_{\mathrm{e}} \omega}\right)^{2}}{\mathrm{~T}_{\mathrm{i}}}\right), v_{\mathrm{i}}-\text { ionization frequency. }
\end{aligned}
$$

Thus, an expression has been obtained for estimating the concentration of ions within an angle $\Omega$, taking into account the effect on the state of the electronic subsystem of a powerful EMI with appropriate parameters, which takes into account ion loss due to mobility and the process of ambipolar diffusion.

Electron loss is dependent on recombination processes. Let's determine the effect of recombination on the formation of a highly conductive electron channel of fractions depending on the parameters of exposure to EMR. This process is advisable to consider in time, due to the pulsed nature of EMR.

When particles recombine, energy that equals the binding energy of the particles is seen, recombine. In this case, recombination may be twopart, in which the recombination coefficient does not depend on their concentration, or there may be Triple recombination (excess energy is given to the third part), in which the recombination coefficient is proportional to the concentration of particles of the third kind.

Let's consider the case of a single transition, when the energy of the particles, recombine, varies slightly. For this case, in accordance with ${ }^{12}$, the average recombination time is determined by the ratio:

$$
\tau(\varepsilon)=\int_{-\infty}^{\infty} 1-\mathrm{W}(\varepsilon, \mathrm{t}) \partial \mathrm{t},
$$

12 Baliuk N., Kechyev L., Stepanov P. Powerful electromagnetic impulse: affecting electronic facilities and methods of defence. Moscow. 2007. 478 p. 
where $\mathrm{W}(\varepsilon, \mathrm{t})$ - Immunity of the state of the system, which was in a state with energy $\varepsilon$.

The recombination time can be determined by the ratio [11]:

$$
\tau(\varepsilon)=\frac{2 \mathrm{~T}^{3 / 2}}{\pi^{3 / 2} \mathrm{e}^{6} \mathrm{n}_{\mathrm{i}}} \int_{0}^{\infty} \mathrm{e}^{-\mid \varepsilon / \mathrm{T}}|\varepsilon|^{5 / 2} \mathrm{~d}|\varepsilon| \mathrm{B}^{-1}(\varepsilon),
$$

where $I_{u}-$ ionization potential;

$\rho=\frac{\partial \mathrm{n}}{\partial \mathrm{t}}-$ density of a state at recombination of an electron and an ion;

$\mathrm{T}$ - temperature of recombined particles;

$\mathrm{n}_{\mathrm{i}}$ - ion concentration.

If triple recombination occurs, then to determine the recombination time, it is necessary to find the value $\mathrm{B}(\varepsilon)=\frac{1}{2} \frac{\overline{\partial \Delta \varepsilon}^{2}}{\partial \mathrm{t}}$.

For finding $B(\varepsilon)$ let's suppose that $I_{u}>>T_{e}$.

If an electron moves in the field of an ion according to the Classical law and as a result of a collision with atoms, energy is exchanged, then in accordance with let's write ${ }^{13}$ :

$$
\mathrm{B}(\varepsilon)=\frac{1}{3} \mathrm{~V}_{\mathrm{a}} \mathrm{m}_{\mathrm{e}}^{2} \mathrm{~V}_{\mathrm{e}}^{2} \mathrm{n}_{\mathrm{a}} \sigma,
$$

where $\mathrm{V}_{\mathrm{a}}$ - atom velocity with which the electron exchanges energy;

$\mathrm{V}_{\mathrm{e}}$ - electron velocity;

$\mathrm{n}_{\mathrm{a}}$ - concentration of atoms;

$\sigma$-diffuse section of elastic scattering of an electron on an atom.

On average, this expression is the velocity of atoms and electrons. Then for the coordinate system where the ion is stationary, let's write:

$$
\left\langle\mathrm{V}_{\mathrm{a}}^{2}\right\rangle=\frac{3 \mathrm{~T}_{A}}{\mathrm{~m}_{\mathrm{a}}}+\mathrm{V}_{\mathrm{i}}^{2},
$$

where $\mathrm{T}_{\mathrm{A}}-$ air temperature; $\mathrm{m}_{\mathrm{a}}-$ atom mass.

13 Alybin V.G. Problems of creation of protective devices for microwave radar and communication. 12th Int. Crimean Conference "Microware Telecommunication Technology”. (Sevastopol, 9-13 September. 2002). Sevastopol. 2002. Pp. 15-21. 
At an insignificant electron velocity $\sigma=$ const. Then for the bound electron let's obtain ${ }^{14}$ :

$$
\overline{\mathrm{V}}_{\mathrm{e}}^{3}=\frac{16}{3 \pi}\left(\frac{2|\varepsilon|}{\mathrm{m}_{\mathrm{e}}}\right)^{3 / 2} .
$$

From (36) for $\varepsilon<0$ let's find:

$$
\mathrm{B}(\varepsilon)=\frac{\mathrm{T}_{A}}{\mathrm{~m}_{\mathrm{a}}}\left(1+\frac{\mathrm{m}_{\mathrm{a}} \mathrm{V}_{\mathrm{i}}^{2}}{3 \mathrm{~T}_{\mathrm{\Pi}}}\right) \mathrm{n}_{\mathrm{a}} \sigma \mathrm{m}_{\mathrm{e}}^{1 / 2} \frac{16}{3 \pi}(2|\varepsilon|)^{3 / 2} .
$$

Taking into account (37), let's obtain the expression for the recombination time:

$$
\tau_{\mathrm{p}}=\frac{3 \mathrm{~m}_{\mathrm{a}} \mathrm{T}_{\mathrm{e}}^{7 / 2}}{16 \sqrt{2 \pi \mathrm{e}^{6}} \mathrm{n}_{\mathrm{i}} \mathrm{T}_{A}\left(1+\frac{\mathrm{m}_{\mathrm{a}} \mathrm{V}_{\mathrm{i}}^{2}}{3 \mathrm{~T}_{A}}\right) \mathrm{n}_{\mathrm{a}} \sigma \mathrm{m}_{\mathrm{e}}^{1 / 2}} .
$$

The recombination coefficient averaged over the ion velocities is determined in accordance with the ratio:

$$
\alpha_{\mathrm{p}}=\frac{1}{\tau_{\mathrm{p}} \mathrm{n}_{\mathrm{i}}}-\frac{16 \sqrt{2 \pi}}{3} \frac{\mathrm{e}^{6} \mathrm{n}_{\mathrm{a}}}{\mathrm{T}_{\mathrm{e}}^{7 / 2}} \frac{\mathrm{T}_{A} \sigma \sqrt{\mathrm{m}_{\mathrm{e}}}}{\mu_{r}},
$$

where $\mu_{r}$-reduced mass of the atom and the ion.

At atmospheric pressure in air, the coefficient of ion-ion recombination is $\alpha_{\mathrm{p}} \approx 2.4 \times 10^{-6} \frac{\mathrm{sm}}{\mathrm{s}}$.

Electron-ion recombination has a much lower probability for the high velocity of electrons, limiting the time of interaction of particles and the capture of an electron to form a neutral particle.

Thus, the recombination of charged particles at different atmospheric pressure and air temperature leads to a decrease in the initial concentration of electrons in the discharge gap, which, in turn, will lead to an increase in the time of occurrence of a highly conductive channel and under the influence of a large amount of EMR energy, which may exceed acceptable level.

${ }^{14}$ Alybin V.G. Problems of creation of protective devices for microwave radar and communication. 12th Int. Crimean Conference "Microware Telecommunication Technology”. (Sevastopol, 9-13 September. 2002). Sevastopol. 2002. Pp. 15-21. 


\section{CONCLUSIONS}

1. The diffusion coefficient for a weakly ionized medium is determined, which is almost twice the ion diffusion coefficient, reduces the electronic conductivity of the medium and, accordingly, negatively affects the implementation of the EMR circuit when it interacts with the REM.

2. It is established that the CCD EMR has a significant effect, namely, the ion mobility with a high electric field voltage depends inversely on the voltage of this field and does not depend on the electric field with a small voltage, which characterizes the operating signal of the REM and, accordingly, on the formation of a highly conductive channel for the implementation of the closure of EMR in the means of REM protection.

3. Expressions are obtained for estimating the concentration of ions within an angle $\Omega$, taking into account the effect on the state of the electronic subsystem of powerful EMR with appropriate parameters, which takes into account ion loss due to mobility and the process of ambipolar diffusion.

4. Recombination of charged particles at different values of atmospheric pressure and air temperature leads to a decrease in the initial concentration of electrons in the discharge gap, which, in turn, leads to an increase in the time of occurrence of a highly conductive channel and under the influence of a higher amount of EMR energy, which may exceed the maximum allowable level.

\section{SUMMARY}

The subject of this research is determination of the loss of charged particles in the pre-ionized air medium of the openings of the screen housings of radio-electronic means (REM) and waveguide antenna inputs depending on the parameters of electromagnetic radiation (EMR). The aim of research is establishment of the dependence of the diffusion coefficients, sticking, electron temperature and recombination on the parameters of the electromagnetic radiation, which determine the loss of electrons during the formation of a highly conductive channel in the discharge gap. The need to determine the loss of charged particles when solving the problem of protecting the REM from the effects of powerful electromagnetic radiation based on the use of nature-friendly (plasma) technologies is due to the need to determine the time of formation of the high-conductive channel and the strength of the electromagnetic radiation at which the breakdown in the discharge gap will be carried 
out. The article solves the following tasks: analysis of known scientific results in the field of development and research of the interaction of ionized air with EMR; determination of the influence of the working signal on the diffusion and mobility of ions; determination of the influence of high-frequency (HF) and microwave (MW) electric field on diffusion, mobility, electron temperature of charged particles of a weakly ionized air medium in an orifice (antenna waveguide) to obtain an analytical expression for estimating the concentration of charged particles within the scattering angle taking into account the effect on the state of the electronic subsystem powerful EMR; determination of the EMR effect on the recombination of charged particles during the formation of a highly conductive electron channel.

\section{REFERENCES}

1. Benford J., Swegle J. High-Power Microwave. Norxood. 1991. 412 p.

2. Kravchenko V.I. Elektromagnitnoe oruzhie. Kharkov. 2008. 185 p.

3. Dobykin V.D., Kupriyanov A.I., Ponomarev V.G., Shustov L.N. Electronic Warfare. Power failure of electronic systems. Moscow. 2007. 487 p.

4. Starostenko V.V., Grigoriev V.V., Taran E.P. The impact of electromagnetic fields on the stability of the IC. Mat. of 6th Int. Crimean Microwave Conf. (Sevastopol, Sept. 16-19. 1996). Sevastopol. 1996. Pp. 188-191.

5. Artsimovich L.A., Sagdeev R.Z. Plasma physics for physicists. Moscow. 1979. 320 p.

6. Myrova L.O., Chepizhenko A.Z. Ensuring stability of communications equipment to the ionizing and electromagnetic radiation. Moscow. 1988. 296 p.

7. Magda I.I., Bludov S.P., Gadetski N.P., et al. Studies on electronic device degradation phenomena under interference of pulsed-power electromagnetic fields. 3-rd Int. Crimean Conf. "UNF Tech. and satellite reception”. Sevastopol. 1993. Pp. 523-526.

8. Vorobiov O., Savchenko V., Sotnikov A., Tarshin V., Kurtseitov T. Development of radioisotopic-plasmatechnology for the protection of radio electronic means from powerful electromagnetic radiation. Eastern-European Journal of Enterprise Technologies. 2017. Vol. 1, No. 5(85). Pp. 16-22. DOI: 10.15587/1729-4061.2017.91642. 
9. Krutov A., Mitmen V., Rebrov A. Protector of the small power level. Materials proc. of 12th International Crimean Conference “Microwave equipment”. Sevastopol. 2002. Pp. 93-94.

10. Ricketts L.U., Bridges J.J. Mayletta electromagnetic pulse and methods of protection. Moscow. 1979. 328 p.

11. Alybin V.G. Problems of creation of protective devices for microwave radar and communication. 12th Int. Crimean Conference "Microware Telecommunication Technology". (Sevastopol, 9-13 September. 2002). Sevastopol. 2002. P. 15-21.

12. Ropy A.N., Starik A.M., Shutov K.K. Microwave protective device. Moscow. 1993. 128 p.

13. Krizny A.V., Vorobyov O.M., Sotnikov O.M. Designing the structure of the material of the protective screens of radio-electronic means of arms and military equipment from the effects of powerful electromagnetic radiation pulse duration. Trudy Universitetu. 2013. No. 6 (120). Pp. 187-191.

14. Baliuk N., Kechyev L., Stepanov P. Powerful electromagnetic impulse: affecting electronic facilities and methods of defence. Moscow. 2007. $478 \mathrm{p}$.

\section{Information about authors:}

Iasechko M. M.,

Doctor of Engineering Sciences,

Professor at the Department of Air Defense Armaments of the Land Forces

Ivan Kozhedub Kharkiv National Air Force University 77/79, Sumska str., Kharkiv, 61000, Ukraine

Smyrnova I. M., Doctor of Pedagogic Sciences, Deputy Director for Scientific and Pedagogical Work Danube Institute of National University “Odessa Maritime Academy” 9, Fanahoriiska str., Izmail, Odessa region, 68607, Ukraine 